\title{
CHOW COHOMOLOGY GROUPS OF ALGEBRAIC SURFACES
}

\author{
MASAKI HANAMURA
}

\begin{abstract}
For a normal algeraic surface we consider its higher Chow groups and Chow cohomology (the latter defined by the author). We study the canonical map from the Chow cohomology to the higher Chow group of the surface by relating it to the canonical map from the higher Chow group to Chow cohomology of the exceptional divisor of a desingularization of the surface.
\end{abstract}

\section{Introduction}

For a quasi-projective variety $S$ over a field, S. Bloch defined its higher Chow groups $\mathrm{CH}^{r}(S, n)$ as the homology of a certain complex $Z^{r}(S, \cdot)$ called the cycle complex, $[\mathrm{Bl} 1, \mathrm{Bl} 2, \mathrm{Bl} 3]$. One may view this as a Borel-Moore homology theory; for example it is covariantly functorial for proper maps, and contravariantly functorial for open immersions (more generally for flat maps).

For a quasi-projective variety $S$ over a field of characteristic zero, using resolution of singularities and Bloch's cycle complexes, we defined the Chow cohomology groups $\mathrm{CHC}^{r}(S, n)$ (see [Ha 2] for details). To briefly recall it, we take a cubical hyperresolution $X_{\bullet} \rightarrow S$, which gives a strict truncated simplicial scheme; it consists of smooth varieties $X_{a}$ for $0 \leq a \leq N$ with some $N$, and the face maps $d_{i}: X_{n} \rightarrow X_{n-1}$, $i=0, \ldots, n$, satisfying the usual identities. (It differs from a simplicial scheme in that there are only face maps and no degeneracies, and there are only finitely many terms.) See $[$ GNPP] for details on cubical hyperresolutions.

We then form the double complex

$$
Z^{r}\left(X_{0}, \cdot\right) \stackrel{d^{*}}{\longrightarrow} Z^{r}\left(X_{1}, \cdot\right) \stackrel{d^{*}}{\longrightarrow} \cdots \stackrel{d^{*}}{\longrightarrow} Z^{r}\left(X_{N}, \cdot\right)
$$

where the ath column is the cycle complex of $X_{a}$, and the horizontal differentials $d^{*}: z^{r}\left(X_{a}, \cdot\right) \rightarrow Z^{r}\left(X_{a+1}, \cdot\right)$ are the alternating sums of the pull-backs $d_{i}^{*}$ by the face maps. (Strictly speaking one must take appropriate quasi-isomorphic subcomplexes for $d^{*}$ be defined, see Section 1.) The total complex of this double complex is denoted $Z^{r}\left(X_{\bullet}, \cdot\right)^{*}$, and called the cohomological cycle complex of $S$. Then $\operatorname{CHC}^{r}(S, n)$ is by definition the $(-n)$ th cohomology of $Z^{r}\left(X_{\bullet}, \cdot\right)^{*}$.

It is proven in [Ha 2] that $\mathrm{CHC}^{r}(S, n)$ is well-defined up to canonical isomorphism, independent of the choice of a hyperresolution, and that the association $S \mapsto$ $\mathrm{CHC}^{r}(S, n)$ is contravariantly functorial for all maps. The condition on the characteristic is unnecessary if $\operatorname{dim} S \leq 2$, since its desingularization (and thus its cubical hyperresolution) exist. In that case we exhibit the cubical hyperresolution and the cohomological cycle complex of $S$ explicitly in Sections 1 and 2. (The relationship of

Received by the editors August 15, 2011. 
our Chow cohomology to the motivic cohomology of Friedlander and Voevodsky [FV] will be discussed in a separate paper.)

If $d=\operatorname{dim} S$, there is a canonical map $\mathrm{CHC}^{r}(S, n) \rightarrow \mathrm{CH}_{d-r}(S, n)$; it is an isomorphism if $S$ is smooth. This is analogous to the following situation in topology. For a good topological space $S$, there is a canonical map from cohomology to Borel-Moore homology, $H^{i}(S) \rightarrow H_{2 \operatorname{dim} S-i}^{B M}(S)$, induced by the cap product by the fundamental class. The purpose of this paper is to study this map (tensored with $\mathbb{Q}$ ) for a normal surface $S$. We take a desingularization $p: X \rightarrow S$ so that the exceptional locus $E$ is a divisor with normal crossings. We show in (3.3).

Theorem. Let $S$ be a normal quasi-projective surface over an algebraically closed field of characteristic zero. Then the canonical map $\mathrm{CHC}^{r}(S, n)_{\mathbb{Q}} \rightarrow \mathrm{CH}_{2-r}(S, n)_{\mathbb{Q}}$ is an isomorphism for all $r, n$ if and only if $E$ is a rational tree.

The paper is organized as follows. In Section 1, we give some calculations of the Chow cohomology of curves, in particular of normal crossing divisors on a smooth surface. In Section 2, we describe the Chow cohomology and homology of a normal surface $S$ in terms of the Chow cohomology and homology of its desingularization $X$ and the exceptional divisor $E$. We prove the above theorem in Section 3 ; the canonical map from the Chow cohomology to homology of $S$ is studied by relating it to the canonical map from the Chow homology to cohomology of the exceptional divisor.

In this paper, we consider quasi-projective varieties over a field $k$. Although the Chow cohomology and homology can be defined for a variety over any field (if the characteristic is zero), for explicit computations for curves and surfaces we assume $k$ to be algebraically closed.

We point out some prior work on the subject. Vistoli studied varieties for which the operational Chow groups (as in $[\mathrm{Fu}]$ ) are isomorphic to the Chow groups, [Vi]. Subsequently Kimura worked on operational Chow groups and hyper-envelopes, [Ki]. The main difference from the present work is that they take the operational theory while we consider the Chow cohomology. But the reader also observes similarities to our approach: we use desingularizations and hyperresolutions instead of hyperenvelopes.

\section{Chow cohomology of curves}

We consider quasi-projective varieties over a field $k$. We refer to [Bl 1, Bl 2, Bl 3] for the details of the theory of higher Chow groups. Here we recall the basic properties.

(1) Let $\square^{1}=\mathbb{P}_{k}^{1}-\{1\}$ and $\square^{n}=\left(\square^{1}\right)^{n}$ with coordinates $\left(x_{1}, \ldots, x_{n}\right)$. Faces of $\square^{n}$ are intersections of codimension one faces, and the latter are divisors of the form $\square_{i, a}^{n-1}=\left\{x_{i}=a\right\}$ where $a=0$ or $\infty$. A face of dimension $m$ is canonically isomorphic to $\square^{m}$.

Let $X$ be an equi-dimensional variety (or a scheme). Let $Z^{r}\left(X \times \square^{n}\right)$ be the free abelian group on the set of codimension $r$ irreducible subvarieties of $X \times \square^{n}$ meeting each $X \times$ (face) properly. An element of $Z^{r}\left(X \times \square^{n}\right)$ is called an admissible cycle. 
The inclusions of codimension one faces $\delta_{i, a}: \square_{i, a}^{n-1} \hookrightarrow \square^{n}$ induce the map

$$
\partial=\sum(-1)^{i}\left(\delta_{i, 0}^{*}-\delta_{i, \infty}^{*}\right): \mathcal{Z}^{r}\left(X \times \square^{n}\right) \rightarrow Z^{r}\left(X \times \square^{n-1}\right) .
$$

One has $\partial \circ \partial=0$. Let $\pi_{i}: X \times \square^{n} \rightarrow X \times \square^{n-1}, i=1, \ldots, n$ be the projections, and $\pi_{i}^{*}: Z^{r}\left(X \times \square^{n-1}\right) \rightarrow Z^{r}\left(X \times \square^{n}\right)$ be the pull-backs. Let $Z^{r}(X, n)$ be the quotient of $Z^{r}\left(X \times \square^{n}\right)$ by the sum of the images of $\pi_{i}^{*}$. Thus an element of $Z^{r}(X, n)$ is represented uniquely by a cycle whose irreducible components are non-degenerate (not a pull-back by $\left.\pi_{i}\right)$. The map $\partial$ induces a map $\partial: Z^{r}(X, n) \rightarrow Z^{r}(X, n-1)$, and $\partial \circ \partial=0$. The complex $Z^{r}(X, \cdot)$ thus defined is the cycle complex of $X$ in codimension $r$. The higher Chow groups are the homology groups of this complex:

$$
\mathrm{CH}^{r}(X, n)=H_{n} z^{r}(X, \cdot)
$$

Note that $\mathrm{CH}^{r}(X, 0)=\mathrm{CH}^{r}(X)$, the Chow group of $X$. In this paper, we would rather use the indexing by dimensions: for $s \in \mathbb{Z}, Z_{s}(X, \cdot)=Z^{\operatorname{dim} X-s}(X, \cdot)$, and $\mathrm{CH}_{s}(X, n)$ is the homology group.

(2) For a proper map $f: X \rightarrow Y$ of $k$-schemes, the push-forward $f_{*}: z_{s}(X, \cdot) \rightarrow$ $Z_{s}(Y, \cdot)$, hence also $f_{*}: \mathrm{CH}_{s}(X, n) \rightarrow \mathrm{CH}_{s}(Y, n)$ is defined.

(3) For a flat map $f: X \rightarrow Y$ of relative equi-dimension $d$, the pull-backs $f^{*}$ : $z_{s}(Y, \cdot) \rightarrow z_{s+d}(X, \cdot)$ and $f^{*}: \mathrm{CH}_{s}(Y, n) \rightarrow \mathrm{CH}_{s+d}(X, n)$ are defined. If $f: X \rightarrow Y$ is a map where $Y$ is smooth and $X$ equi-dimensional, there is a map $f^{*}: \mathrm{CH}^{r}(Y, n) \rightarrow \mathrm{CH}^{r}(X, n)$. In fact, there is a quasi-isomorphic subcomplex $Z^{r}(Y, \cdot)^{\prime}$ of $Z^{r}(Y, \cdot)$ on which $f^{*}: Z^{r}(Y, \cdot)^{\prime} \rightarrow Z^{r}(X, \cdot)$ is defined.

(4) If $X$ is smooth quasi-projective and equi-dimensional, one has the intersection product $\mathrm{CH}_{s}(X, n) \otimes \mathrm{CH}_{t}(X, m) \rightarrow \mathrm{CH}_{s+t-\operatorname{dim} X}(X, n+m)$.

(5) Projection formula.

(6) Projective bundle formula.

(7) Localization sequence. If $X$ is a quasi-projective variety and $U$ is an open set, letting $Z=X-U$, one has an exact sequence of complexes $0 \rightarrow z_{s}(Z, \cdot) \rightarrow$ $Z_{s}(X, \cdot) \rightarrow Z_{s}(U, \cdot)$. The localization theorem $[\mathrm{Bl} 2]$ asserts that the induced $\operatorname{map} z_{s}(X, \cdot) / z_{s}(Z, \cdot) \rightarrow z_{s}(U, \cdot)$ is a quasi-isomorphism.

(8) The self-intersection formula [Ha 3, Section 1].

Let $X$ be a smooth quasi-projective variety and $Y \subset X$ a smooth closed subvariety of codimension $d, i: Y \rightarrow X$ the closed immersion, and $N=N_{Y} X$ the normal bundle. Then

$$
i^{*} i_{*}(y)=c_{d}(N) \cdot y
$$

for $y \in \mathrm{CH}_{s}(Y, n)$.

In this paper, we need this only for $X$ smooth projective; in that case this can also be deduced from the formula $i^{*} \circ i_{*}=c_{d}(N)$ in the correspondence ring $\mathrm{CH}^{\operatorname{dim} Y}(Y \times Y)$ (see $[\mathrm{Ma}])$. 
The notion of cubical hyperresolution is given in [GNPP]. We review it to the extent that will be sufficient for our purposes.

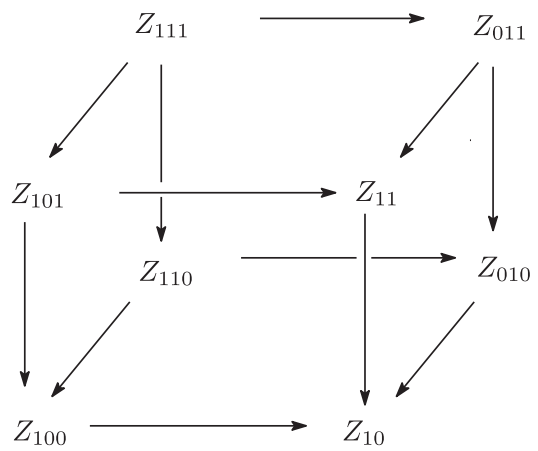

$(1-2)$

We first explain the notion of 2-resolution. Let $S$ be a quasi-projective variety. A commutative square

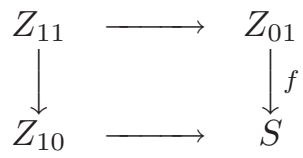

is called a 2-resolution if it satisfies the following conditions:

(1) The varieties $Z_{a b}$ are reduced, and the square is Cartesian up to taking the reduced scheme structure (namely, $Z_{11}$ is isomorphic to the reduced scheme of $\left.Z_{10} \times{ }_{S} Z_{01}\right)$.

(2) $Z_{01}$ is smooth.

(3) The horizontal arrows are closed immersions.

(4) $f$ is a proper map.

(5) $f$ induces an isomorphism over $S-Z_{10}$.

If in addition $Z_{10}$ and $Z_{11}$ are also smooth, the diagram $Z_{\bullet \bullet}$ gives a cubical hyperresolution; note that the square has $S$ at the end vertex, and smooth varieties at the other vertices. For example if $S$ is a curve, there is such a 2-resolution. The associated strict simplicial scheme $X_{\bullet}$ is given by

$$
X_{n}=\coprod_{\alpha_{0}+\alpha_{1}=n+1} Z_{\alpha_{0} \alpha_{1}}
$$

for $n=0,1$, namely by $X_{0}=Z_{01} \coprod Z_{10}, X_{1}=Z_{11}$; the face maps $d_{i}: X_{1} \rightarrow X_{0}$ are the obvious ones. There is also the augmentation map $X_{\bullet} \rightarrow S$.

In general, $Z_{10}$ and $Z_{11}$ are not smooth; then one must take 2-resolutions of $Z_{10}$ and $Z_{11}$ in a manner compatible with the map $Z_{11} \rightarrow Z_{10}$, and form the following commutative (3-dimensional) cube diagram, with the map $Z_{11} \rightarrow Z_{10}$ on an edge. The top face gives a 2 -resolution of $Z_{11}$, and the bottom face gives one for $Z_{10}$. If $\operatorname{dim} S \leq 2$, which is the case we will be interested in, one can take such a diagram with all the varieties at the corners, except $Z_{11}$ and $Z_{10}$, smooth. In that case by "glueing" the cube (1-2) with the 2-resolution (1-1) along the edge $Z_{11} \rightarrow Z_{10}$, we obtain the following cube diagram, with $S$ at the end corner, and with all the other 
varieties smooth.

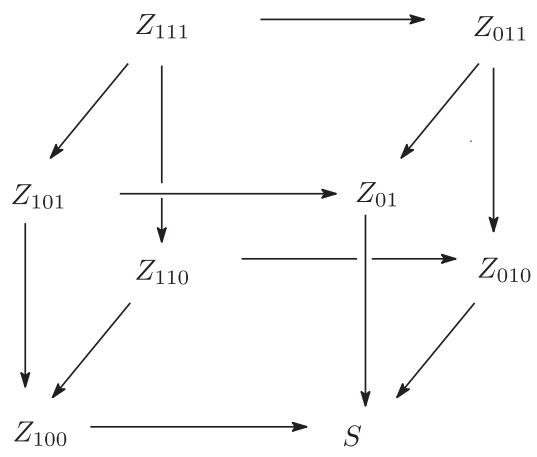

By definition, this cube digram is a cubical hyperresolution of $S$. The associated strict simplicial scheme $X_{\bullet}$ is given by

$$
X_{n}=\coprod_{\alpha_{0}+\alpha_{1}+\alpha_{2}=n+1} Z_{\alpha_{0} \alpha_{1} \alpha_{2}}
$$

for $n=0,1,2$, with the obvious face maps.

For a variety $S$ of higher dimension, one must iterate this process a finite number of times to arrive at cubical hyperresolution. See [GNPP] for details.

We next recall the definition of Chow cohomology groups for curves. For a quasiprojective curve over $k$, not necessarily irreducible, let $p: \tilde{C} \rightarrow C$ be the normalization of $C, \Sigma \subset C$ the singular locus, and $\tilde{\Sigma}=p^{-1}(\Sigma)$ (with reduced scheme structure). One has a commutative square

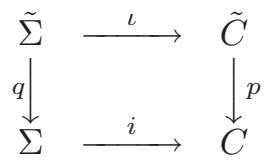

where $i$ is the embedding and $\iota, q$ are the induced maps. This gives a cubical hyperresolution of $C$. Let

$$
Z^{r}(C, \cdot)^{*}:=\text { Cone }\left[Z^{r}(\tilde{C}, \cdot) \oplus Z^{r}(\Sigma, \cdot) \stackrel{\iota^{*}-q^{*}}{\longrightarrow} Z^{r}(\tilde{\Sigma}, \cdot)\right][-1] .
$$

To be precise one must replace $Z^{r}(\tilde{C}, \cdot)$ with a quasi-isomorphic subcomplex so that the pull-back $\iota^{*}: Z^{r}(\tilde{C}, \cdot) \rightarrow Z^{r}(\tilde{\Sigma}, \cdot)$ is defined. The cycle complex is a homological complex, which can be viewed as a cohomological complex in the usual manner. So $Z^{r}(C, \cdot)^{*}$ is a cohomological complex. We let

$$
\mathrm{CHC}^{r}(C, n)=H^{-n} Z^{r}(C, \cdot)^{*} .
$$

We also write $\mathrm{CHC}^{r}(C)=\mathrm{CHC}^{r}(C, 0)$. There is a long exact sequence

$$
\begin{array}{cccccc}
\rightarrow & \mathrm{CHC}^{r}(C, n) & \rightarrow & \mathrm{CH}^{r}(\tilde{C}, n) \oplus \mathrm{CH}^{r}(\Sigma, n) & \rightarrow & \mathrm{CH}^{r}(\tilde{\Sigma}, n) \\
& \rightarrow & \mathrm{CHC}^{r}(C, n-1) & \rightarrow & \cdots .
\end{array}
$$

As is obvious from the definition, if $C^{\prime} \rightarrow C$ is the semi-normalization, $C^{\prime}$ and $C$ have the same higher Chow cohomology. For $r=1$, the following can be shown using the fact that if $X$ is smooth $\mathrm{CH}^{1}(X, n)=0$ for $n \neq 0,1$, and $\mathrm{CH}^{1}(X, 1)=\Gamma\left(X, \mathcal{O}_{X}^{*}\right)$ (see $[\mathrm{Bl} 1])$. 
Proposition 1.1. Let $C$ be a quasi-projective curve over $k$. Then $\operatorname{CHC}^{1}(C, n)=0$ for $n \neq 0,1$, and there is an exact sequence

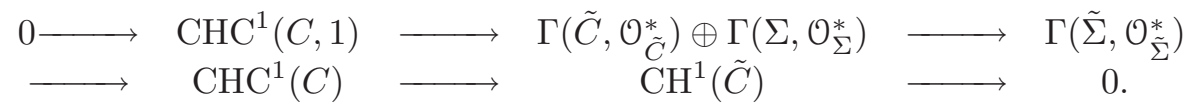

In the rest of this section, we assume $k$ is algebraically closed. Then for $C$ projective and irreducible, the result is much simpler:

Proposition 1.2. Let $C$ be an irreducible projective curve over an algebraically closed field $k$. Then $\mathrm{CHC}^{1}(C, n)=0$ for $n \neq 0,1, \mathrm{CHC}^{1}(C, 1)=k^{*}$, and there is an exact sequence

$$
0 \rightarrow \bigoplus_{P \in \Sigma}\left(\underset{Q \mapsto P}{\bigoplus} k^{*}\right) / k^{*} \rightarrow \mathrm{CHC}^{1}(C) \rightarrow \mathrm{CH}^{1}(\tilde{C}) \rightarrow 0 .
$$

(For each $P$ in $\Sigma$, one takes the direct sum of copies of $k^{*}$, one for each $Q \in \tilde{C}$ over $P$, and mod out by the subgroup $k^{*}$ embedded diagonally in the sum.)

Proof. This follows from Proposition 1.1 and the identities $\Gamma\left(\tilde{C}, \mathcal{O}_{\tilde{C}}^{*}\right)=k^{*}$,

$$
\Gamma\left(\Sigma, \mathcal{O}_{\Sigma}^{*}\right)=\bigoplus_{P \in \Sigma} k^{*}, \quad \text { and } \quad \Gamma\left(\tilde{\Sigma}, \mathcal{O}_{\tilde{\Sigma}}^{*}\right)=\bigoplus_{Q \in \tilde{\Sigma}} k^{*}
$$

Let $S$ be a smooth quasi-projective surface on a field $k$, and $E$ be a connected normal crossing divisor, with each irreducible component projective, on $S$. Let $\left\{E_{i}\right\}_{i=1, \ldots, N}$ be the irreducible components of $E$, and $E_{i j}=E_{i} \cap E_{j}$ for $i<j$ be the points of intersection. Set $E^{(0)}:=\coprod E_{i}, E^{(1)}:=\coprod_{i<j} E_{i j}$. Let

$$
\delta_{i}: E^{(1)} \rightarrow E^{(0)}, \quad i=0,1
$$

be the maps which restrict to the inclusions $E_{i j} \rightarrow E_{j}, E_{i j} \rightarrow E_{i}$, respectively. There is a natural map $a: E^{(0)} \rightarrow E$. The cohomological cycle complex of $E$ is quasiisomorphic to the complex

$$
Z^{r}(E, \cdot)^{*}=\operatorname{Cone}\left[Z^{r}\left(E^{(0)}, \cdot\right) \stackrel{\delta^{*}}{\longrightarrow} Z^{r}\left(E^{(1)}, \cdot\right)\right][-1]
$$

where $\delta^{*}=\delta_{0}^{*}-\delta_{1}^{*}$. Indeed a cubical hyperresolution of $E$ is given by the Cartesian square

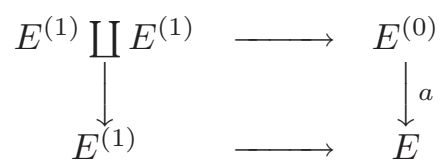

where the inverse image of $E^{(1)}$ by $a$ is identified with $E^{(1)} \amalg E^{(1)}$. The associated cohomological cycle complex is obviously quasi-isomorphic to the complex just mentioned.

The dual graph $\Gamma$ of $E$ consists of the vertices corresponding to the components $E_{i}$, and the edges corresponding to $E_{i j}$. Since the vertices are ordered, the edges are oriented. Also, $\Gamma$ is connected since $E$ is connected.

The chain complex of $\Gamma$ is the two term homological complex

$$
C_{\bullet}(\Gamma)=\left[C_{1}(\Gamma) \stackrel{\partial}{\longrightarrow} C_{0}(\Gamma)\right]
$$


where $C_{0}(\Gamma)$ (resp. $\left.C_{1}(\Gamma)\right)$ is a free $\mathbb{Z}$-module with basis $\left\{E_{i}\right\}$ (resp. $\left\{E_{i j}\right\}$ ), and $\partial$ sends $E_{i j}$ to $E_{j}-E_{i}$. The homology of this complex is denoted by $H_{*}(\Gamma)$. One has $H_{0}(\Gamma)=\mathbb{Z}$ since $\Gamma$ is connected, and $H_{1}(\Gamma)$ is a finitely generated free abelian group.

The cochain complex $C^{\bullet}(\Gamma)$ is the two term complex $d: C^{0}(\Gamma) \rightarrow C^{1}(\Gamma)$ where $C^{i}(\Gamma)=\operatorname{Hom}\left(C_{i}(\Gamma), \mathbb{Z}\right)$ and $d$ is the dual of $\partial$ with minus sign. (The change of sign is made so that the natural evaluation map is a map of complexes.) So $C^{0}(\Gamma)$ (resp. $C^{1}(\Gamma)$ ) is the free $\mathbb{Z}$-module with basis $\left\{e_{i}\right\}$ dual to $\left\{E_{i}\right\}$ (resp. with basis $\left\{e_{i j}\right\}$ dual to $\left.\left\{E_{i j}\right\}\right)$, and

$$
d\left(e_{i}\right)=\sum_{i<j} e_{i j}-\sum_{m<i} e_{m i} .
$$

By definition $H^{*}(\Gamma)$ is the cohomology of this complex. We have $H^{0}(\Gamma)=\mathbb{Z}$ and $H^{1}(\Gamma)=\operatorname{Hom}\left(H_{1}(\Gamma), \mathbb{Z}\right)$ by the universal coefficient theorem.

We say $E$ is a rational tree if each $E_{i}$ is isomorphic to $\mathbb{P}^{1}$ and $H_{1}(\Gamma)=0$ (equivalently, $\left.H^{1}(\Gamma)=0\right)$.

Proposition 1.3. For a connected normal crossing divisor $E$ as above, we have $\operatorname{CHC}^{1}(E, n)=0$ if $n \neq 0,1, \operatorname{CHC}^{1}(E, 1)=k^{*}$, and there is an exact sequence

$$
0 \rightarrow H^{1}(\Gamma) \otimes k^{*} \rightarrow \mathrm{CHC}^{1}(E) \stackrel{a^{*}}{\rightarrow} \mathrm{CH}^{1}\left(E^{(0)}\right) \rightarrow 0 .
$$

Proof. Recall $\mathrm{CHC}^{1}(E, n)$ is the homology of the complex

$$
\text { Cone }\left[Z^{1}\left(E^{(0)}, \cdot\right) \stackrel{\delta^{*}}{\longrightarrow} Z^{1}\left(E^{(1)}, \cdot\right)\right][-1] .
$$

One thus has $\operatorname{CHC}^{1}(E, n)=0$ for $n \neq 0,1$, and an exact sequence

$$
\begin{aligned}
0 & \rightarrow \mathrm{CHC}^{1}(E, 1) \rightarrow \mathrm{CH}^{1}\left(E^{(0)}, 1\right) \stackrel{\delta^{*}}{\longrightarrow} \mathrm{CH}^{1}\left(E^{(1)}, 1\right) \\
& \rightarrow \mathrm{CHC}^{1}(E, 0) \rightarrow \mathrm{CH}^{1}\left(E^{(0)}, 0\right) \longrightarrow 0
\end{aligned}
$$

We have $\mathrm{CH}^{1}\left(E^{(0)}, 1\right)=C^{0}(\Gamma) \otimes k^{*}, \mathrm{CH}^{1}\left(E^{(1)}, 1\right)=C^{1}(\Gamma) \otimes k^{*}$ and $\delta^{*}$ is identified with $d \otimes i d: C^{0}(\Gamma) \otimes k^{*} \rightarrow C^{1}(\Gamma) \otimes k^{*}$. The result now follows.

The group $\mathrm{CHC}^{r}(E, n)$ for $\geq 2$ does not allow a simple description in general. But if $E$ is a normal crossing divisor consisting of rational curves, it can be calculated as follows. Before stating it, we define the reduced Chow cohomology of $E$.

For a connected normal crossing divisor on a smooth surface, let $\pi: E \rightarrow p t=$ Spec $k$ be the structure map, and

$$
\tilde{z}^{r}(E, \cdot)^{*}=\operatorname{Cone}\left[\pi^{*}: z^{r}(p t, \cdot) \rightarrow z^{r}(E, \cdot)^{*}\right] .
$$

Its $(-n)$ th cohomology is denoted $\widetilde{\mathrm{CHC}}^{r}(E, n)$.

There is an exact sequence

$$
\begin{array}{ccccc}
\mathrm{CH}^{r}(p t, n) & \stackrel{\pi^{*}}{\longrightarrow} & \mathrm{CHC}^{r}(E, n) & \longrightarrow & \widetilde{\mathrm{CHC}}^{r}(E, n) . \\
\mathrm{CH}^{r}(p t, n-1) & \longrightarrow & \cdots .
\end{array}
$$

For any closed point $x$ of $E, \pi^{*}$ splits by $x^{*}$, so the above long exact sequence splits to short exact sequences.

Let $\tilde{\pi}: E^{(0)} \rightarrow \amalg_{N} p t$ be the map to the disjoint union of $N$ copies of $p t$, which restricts to the structure map on each component $E_{i}$. It induces the map $\tilde{\pi}_{*}: \mathrm{CH}^{r}\left(E^{(0)}, n\right) \rightarrow \mathrm{CH}^{r-1}(p t, n)^{\oplus N}$. 
Consider the map $\pi^{*}: z^{r}(p t, \cdot) \rightarrow z^{r}\left(E^{(0)}, \cdot\right)$, and take its cone, and let its $(-n)$ th cohomology group be $\mathrm{CH}^{r}\left(E^{(0)} \rightarrow p t, n\right)$. (A possible notation $\widetilde{\mathrm{CH}}^{r}\left(E^{(0)}, n\right)$ is avoided here, since one may confuse it with $\oplus \widetilde{\mathrm{CH}}^{r}\left(E_{i}, n\right)$. Also see the remark below.) The commutative diagram

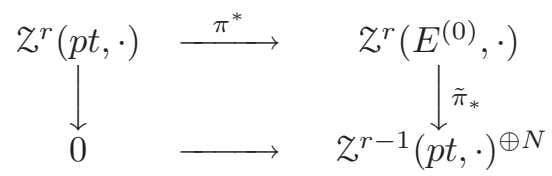

defines a map $\tilde{\pi}_{*}: \mathrm{CH}^{r}\left(E^{(0)} \rightarrow p t, n\right) \rightarrow \mathrm{CH}^{r-1}(p t, n)^{\oplus N}$. From the definitions there is a commutative square

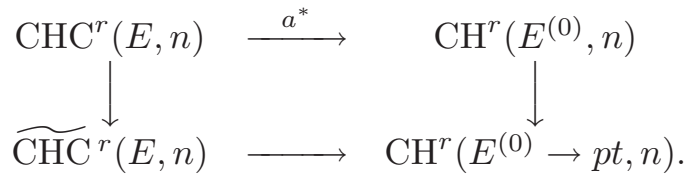

So by composition one defines a map, still called $\tilde{\pi}_{*}$, from any one of the four groups to $\mathrm{CH}^{r-1}(p t, n)^{\oplus N}$.

Remark. For a not necessarily connected normal crossing divisor $E$, let $E_{\lambda}$ be its connected components, define $\tilde{z}^{r}(E, \cdot)^{*}=\oplus_{\lambda} \tilde{z}^{r}\left(E_{\lambda}, \cdot\right)^{*}$, and let $\widetilde{\mathrm{CHC}^{r}}(E, n)=$ $\oplus \widetilde{\mathrm{CHC}}^{r}\left(E_{\lambda}, n\right)$ be its cohomology.

Proposition 1.4. If $E$ is a connected normal crossing divisor with each $E_{i} \cong \mathbb{P}^{1}$, one has an exact sequence ( $N$ is the number of irreducible components of $E$ )

$$
0 \rightarrow H^{1}(\Gamma) \otimes \mathrm{CH}^{r}(p t, n+1) \rightarrow \widetilde{\mathrm{CHC}^{r}}(E, n) \rightarrow \mathrm{CH}^{r-1}(p t, n)^{\oplus N} \rightarrow 0 .
$$

where the surjection is the map $\tilde{\pi}_{*}$. In particular, if $E$ is a rational tree, $\widetilde{\mathrm{CHC}}^{r}(E, n) \cong$ $\mathrm{CH}^{r-1}(p t, n)^{\oplus N}$.

Proof. We have an exact sequence

$$
\begin{array}{cccccc}
\mathrm{CHC}^{r}(E, n) & \longrightarrow & \longrightarrow & \mathrm{CH}^{r}\left(E^{(0)}, n\right) & \stackrel{\delta^{*}}{\longrightarrow} & \mathrm{CH}^{r}\left(E^{(1)}, n\right) \\
& \mathrm{CHC}^{r}(E, n-1) & \longrightarrow & \cdots
\end{array}
$$

Since $\mathrm{CH}^{r}\left(\mathbb{P}^{1}, n\right)=\mathrm{CH}^{r}(p t, n) \oplus \mathrm{CH}^{r-1}(p t, n)$, one has

$$
\mathrm{CH}^{r}\left(E^{(0)}, n\right)=C^{0}(\Gamma) \otimes\left(\mathrm{CH}^{r}(p t, n) \oplus \mathrm{CH}^{r-1}(p t, n)\right),
$$

$\mathrm{CH}^{r}\left(E^{(1)}, n\right)=C^{1}(\Gamma) \otimes \mathrm{CH}^{r}(p t, n) ; \delta^{*}$ is zero on $C^{0}(\Gamma) \otimes \mathrm{CH}^{r-1}(p t, n)$, and coincides with $d \otimes 1$ on $C^{0}(\Gamma) \otimes \mathrm{CH}^{r}(p t, n)$. So we have an exact sequence

$$
0 \rightarrow H^{1}(\Gamma) \otimes \mathrm{CH}^{r}(p t, n+1) \rightarrow \mathrm{CHC}^{r}(E, n) \rightarrow \mathrm{CH}^{r}(p t, n) \oplus \mathrm{CH}^{r-1}(p t, n)^{\oplus N} \rightarrow 0,
$$

proving the proposition.

We will state a result parallel to (1.4) for Chow groups. First we discuss the reduced Chow group. Let

$$
\tilde{z}_{s}(E, \cdot)=\operatorname{Cone}\left[\pi_{*}: z_{s}(E, \cdot) \rightarrow z_{s}(p t, \cdot)\right][-1]
$$


and $\widetilde{\mathrm{CH}}_{s}(E, n)$ be its cohomology. If $E$ is not connected, let $E_{\lambda}$ be its connected components and set

$$
\tilde{z}_{s}(E, \cdot)=\oplus_{\lambda} \tilde{z}_{s}\left(E_{\lambda}, \cdot\right) .
$$

There is a long exact sequence

$$
\begin{array}{lllll}
\widetilde{\mathrm{CH}}_{s}(E, n) & \longrightarrow & \mathrm{CH}_{s}(E, n) \stackrel{\pi_{*}}{\longrightarrow} \mathrm{CH}_{s}(p t, n) \\
\widetilde{\mathrm{CH}}_{s}(E, n-1) & \longrightarrow & \cdots
\end{array}
$$

For any closed point $x$ of $E, \pi_{*}$ splits by $x_{*}$, so the above long exact sequence splits to short exact sequences.

Consider the map $\pi_{*}: z_{s}\left(E^{(0)}, \cdot\right) \rightarrow z_{s}(p t, \cdot)$; take its cone, shift by -1 and denote its $(-n)$ th cohomology by $\mathrm{CH}_{s}\left(E^{(0)} \rightarrow p t, n\right)$. (One should distinguish it from $\mathrm{CH}^{1-s}\left(E^{(0)} \rightarrow p t, n\right)$ defined earlier.) The commutative diagram

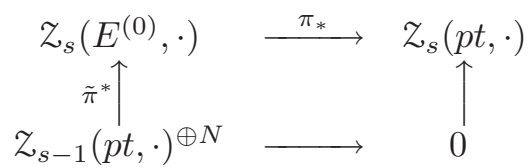

induces a map $\tilde{\pi}^{*}: \mathrm{CH}_{s-1}(p t, n)^{\oplus N} \rightarrow \mathrm{CH}_{s}\left(E^{(0)} \rightarrow p t, n\right)$. There is a commutative square

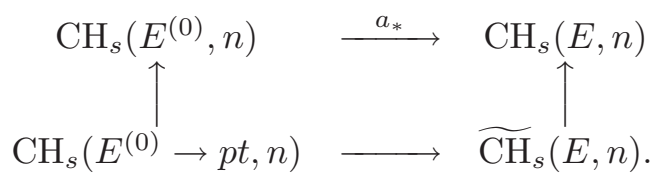

One thus has a map, still denoted $\tilde{\pi}^{*}$, from $\mathrm{CH}_{s-1}(p t, n)^{\oplus N}$ to any one of the four groups. The proof of the following is similar to that of (1.4).

Proposition 1.5. Let $E$ be a connected normal crossing divisor with each $E_{i} \cong \mathbb{P}^{1}$. One has an exact sequence

$$
0 \rightarrow \mathrm{CH}_{s-1}(p t, n)^{\oplus N} \rightarrow \widetilde{\mathrm{CH}}_{s}(E, n) \rightarrow \mathrm{CH}_{s}(p t, n-1) \otimes H_{1}(\Gamma) \rightarrow 0,
$$

where the injection is the map $\tilde{\pi}^{*}$.

If $E$ is a rational tree, $\widetilde{\mathrm{CH}}_{s}(E, n) \cong \mathrm{CH}_{s-1}(p t, n)^{\oplus N}$.

\section{Chow cohomology and homology of surfaces}

Let now $S$ be an irreducible normal quasi-projective surface over a field $k$; so the singular locus $\Sigma$ consists of finite number of points. Let $p: X \rightarrow S$ be a desingularization such that the inverse image (with reduced scheme structure) $E=p^{-1}(\Sigma)$ is a normal crossing divisor. Since $S$ is normal, the inverse image of each point of $\Sigma$ is connected. We have a Cartesian diagram

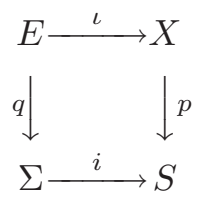

with $i, \iota$ the inclusions and $q$ the induced map. This is the first step of a cubical hyperresolution of $S$. We then construct a cubical hyperresolution of the map $q: E \rightarrow \Sigma$; 
one such is given by a cube where the top square is the square (2-1), and the bottom square the trivial 2-resolution of $\Sigma$, namely four copies of $\Sigma$ with the identity maps. As explained in Section 1, by "glueing" this cube to the 2-resolution of $S$ along the "edge" $q: E \rightarrow \Sigma$, we obtain the following cubical hyperresolution of $S$.

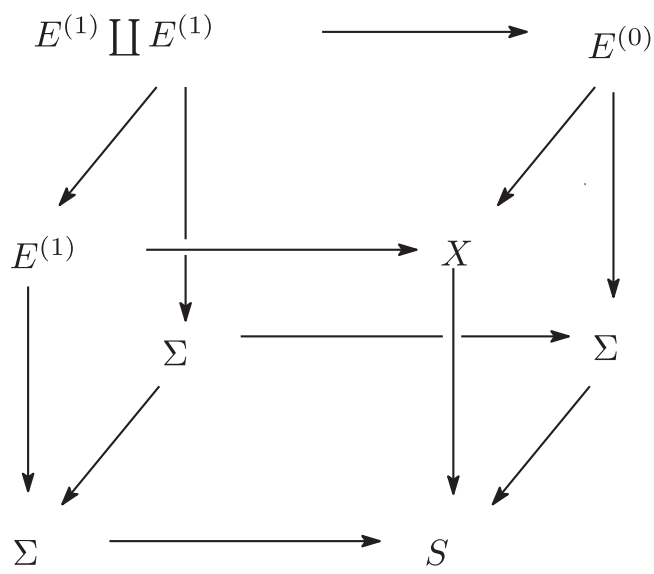

The associated cohomological cycle complex is naturally quasi-isomorphic to the complex defined as

$$
Z^{r}(S, \cdot)^{*}:=\text { Cone }\left[\iota^{*}-q^{*}: Z^{r}(X, \cdot) \oplus Z^{r}(\Sigma, \cdot) \rightarrow Z(E, \cdot)^{*}\right][-1]
$$

with $z^{r}(E, \cdot)^{*}$ defined before. By definition $\operatorname{CHC}^{r}(S, n)$ is the $(-n)$ th cohomology of this complex. In [Ha 2] we showed it is independent of the choice of a desingularization, and contravariantly functorial for all maps. $\operatorname{Note} \mathrm{CHC}^{r}(S, n)=0$ for $n \leq-2$, but $\mathrm{CHC}^{r}(S,-1)$ may not be zero.

There are maps of complexes

$$
Z^{r}(S, \cdot)^{*} \stackrel{p^{*}}{\longrightarrow} Z^{r}(X, \cdot)=z_{2-r}(X, \cdot) \stackrel{p_{*}}{\longrightarrow} z_{2-r}(S, \cdot),
$$

where the first map is the surjection to a quotient. One easily shows that the composition $p_{*} p^{*}$ is independent of the choice of $p: X \rightarrow S$. By passing to homology, one obtains the canonical map $\mathrm{CHC}^{r}(S, n) \rightarrow \mathrm{CH}_{2-r}(S, n)$, namely the composition of the maps

$$
\mathrm{CHC}^{r}(S, n) \stackrel{p^{*}}{\longrightarrow} \mathrm{CH}^{r}(X, n)=\mathrm{CH}_{2-r}(X, n) \stackrel{p_{*}}{\longrightarrow} \mathrm{CH}_{2-r}(S, n) .
$$

In the rest of this section, $k$ is algebraically closed.

Proposition 2.1. We have a long exact sequence

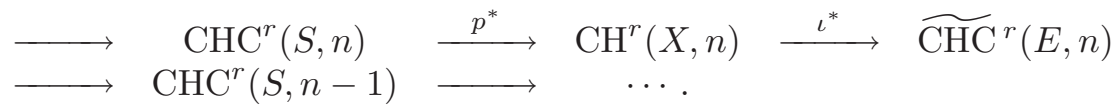

For $r=1$, one has $\mathrm{CHC}^{1}(S, n)=0$ for $n \neq-1,0,1, \mathrm{CHC}^{1}(S, 1)=k^{*}$, and there is an exact sequence

$$
0 \rightarrow \mathrm{CHC}^{1}(S) \stackrel{p^{*}}{\longrightarrow} \mathrm{CH}^{1}(X) \stackrel{\iota^{*}}{\longrightarrow} \mathrm{CHC}^{1}(E) \rightarrow \mathrm{CHC}^{1}(S,-1) \rightarrow 0 .
$$

Proof. The complex $\mathcal{Z}^{r}(S, \cdot)^{*}$ is quasi-isomorphic to

$$
\text { Cone }\left[z^{r}(X, \cdot) \stackrel{\iota^{*}}{\longrightarrow} \frac{z^{r}(E, \cdot)^{*}}{\left.z^{r}(\Sigma, \cdot)\right)}\right][-1] \text {. }
$$


The long exact sequence hence follows. If $r=1$, from (1.3) and the long exact sequence after the definition of $\widetilde{\mathrm{CHC}}^{1}(E, n)$, one deduces the following information on $\widetilde{\mathrm{CHC}}^{1}(E, n): \widetilde{\mathrm{CHC}}^{1}(E, n)=0$ if $n \neq 0$ and $\widetilde{\mathrm{CHC}}^{1}(E)=\mathrm{CHC}^{1}(E)$. The assertion follows from this.

One can study the Chow homology of $S$ using the resolution in a similar manner. As observed in [Ha 2, Section 2], the localization theorem [Bl 3] it follows that if $X_{\bullet} \rightarrow S$ is a cubical hyperresolution, then the complex $z_{s}\left(X_{\bullet}, \cdot\right)_{*}$ defined as the total complex of the double complex

$$
z_{s}\left(X_{N}, \cdot\right) \stackrel{d_{*}}{\longrightarrow} \cdots \stackrel{d_{*}}{\longrightarrow} z_{s}\left(X_{1}, \cdot\right) \stackrel{d_{*}}{\longrightarrow} z_{s}\left(X_{0}, \cdot\right)
$$

is quasi-isomorphic to $z_{s}(S, \cdot)$.

So in our case the complex $z_{s}(S, \cdot)$ is quasi-isomorphic to

$$
\text { Cone }\left[\left(\iota_{*}, q_{*}\right): z_{s}(E, \cdot) \rightarrow z_{s}(X, \cdot) \oplus z_{s}(\Sigma, \cdot)\right]
$$

and thus also to

$$
\text { Cone }\left[\iota_{*}: \tilde{z}_{s}(E, \cdot) \rightarrow z_{s}(X, \cdot)\right]
$$

since $\tilde{z}_{s}(E, \cdot)=$ Cone $\left[q_{*}: z_{s}(E, \cdot) \rightarrow z_{s}(\Sigma, \cdot)\right][-1]$ according to the definition.

Proposition 2.2. We have a long exact sequence

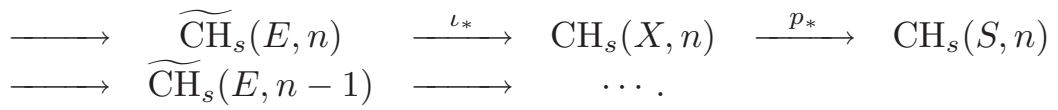

If $s=1$ and $S$ is projective, one has $\mathrm{CH}_{1}(S, n)=0$ if $n \neq 0,1, \mathrm{CH}_{1}(S, 1)=k^{*}$, and there is an exact sequence

$$
0 \rightarrow \mathrm{CH}_{1}(E) \stackrel{\iota_{*}}{\longrightarrow} \mathrm{CH}_{1}(X) \stackrel{p_{*}}{\longrightarrow} \mathrm{CH}_{1}(S) \rightarrow 0
$$

where $\mathrm{CH}_{1}(E)=\bigoplus \mathbb{Z}\left[E_{i}\right]$.

Proof. The exact sequence follows from the definition. If $s=1$, then $\widetilde{\mathrm{CH}}_{1}(E, n)=0$ for $n \neq 0$ and $\widetilde{\mathrm{CH}}_{1}(E)=\mathrm{CH}_{1}(E)$, which is free with generators $E_{i}$. We have an exact sequence

$$
\begin{array}{llllll} 
& 0 \\
& \mathrm{CH}_{1}(E) & \longrightarrow & \mathrm{CH}_{1}(X, 1) & \stackrel{p_{*}}{\longrightarrow} & \mathrm{CH}_{1}(S, 1) \\
\mathrm{CH}_{1}(X) & \longrightarrow & \mathrm{CH}_{1}(S)
\end{array} \longrightarrow 0
$$

where $\mathrm{CH}_{1}(X, 1)=k^{*}$, since $X$ is complete. The map $\mathrm{CH}_{1}(E) \rightarrow \mathrm{CH}_{1}(X)$ is injective since the intersection matrix $\left(E_{i} \cdot E_{j}\right)$ is negative definite, $[\mathrm{Mu}]$. Indeed if $\sum a_{i} E_{i}=0$ in $\mathrm{CH}_{1}(X)$, then the intersection number $\left(\sum a_{i} E_{i}\right)^{2}=0$, so all $a_{i}$ are zero. The assertion now follows.

\section{The map $\mathrm{CHC}^{r}(S, n) \rightarrow \mathrm{CH}_{2-r}(S, n)$}

In this section, assume that $k$ is algebraically closed (although one may take $k$ to be an arbitrary field until (3.3)).

We will consider the higher Chow groups and Chow cohomology groups tensored with $\mathbb{Q}$; for simplicity we will write $\mathrm{CH}_{s}(S, n)$ for $\mathrm{CH}_{s}(S, n)_{\mathbb{Q}}, \mathrm{CHC}^{r}(S, n)$ for $\mathrm{CHC}^{r}(S, n)_{\mathbb{Q}}$, and so on.

Under the same assumption as in the previous section, we study the maps

$$
\mathrm{CH}_{s}(E, n) \stackrel{\iota_{*}}{\longrightarrow} \mathrm{CH}_{s}(X, n)=\mathrm{CH}^{2-s}(X, n) \stackrel{\iota^{*}}{\longrightarrow} \mathrm{CHC}^{2-s}(E, n) .
$$


For this goal, let $X$ be a smooth quasi-projective surface and $E, F$ be smooth projective irreducible curves on $X$; we assume that either $E$ and $F$ meet transversally in a point or $E=F$. We have a Cartesian square

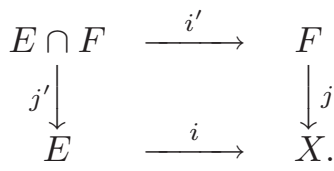

For each $r, n$ one has the map $i^{*} j_{*}: \mathrm{CH}^{r}(F, n) \rightarrow \mathrm{CH}^{r+1}(E, n)$. In case $E \neq F$ this map is induced by the element $\delta_{*}(E \cap F) \in \mathrm{CH}^{2}(E \times F)$; here $\delta: E \cap F \rightarrow F \times E$ is the natural inclusion. Indeed for $\alpha \in \mathrm{CH}^{r}(F)$, we have

$$
\begin{aligned}
\left(\delta_{*}(E \cap F)\right)_{*} \alpha & =p_{1 *}\left[(E \times \alpha) \cdot \delta_{*}(E \cap F)\right] \\
& =p_{1 *} \delta_{*} \delta^{*}(E \times \alpha) \\
& =j_{*}^{\prime} i^{\prime *} \alpha
\end{aligned}
$$

which coincides with $i^{*} j_{*} \alpha$. (The first equality holds by definition, the second by the projection formula and the third by $p_{1} \delta=j^{\prime}$ and $p_{2} \delta=i^{\prime}$.)

If $E=F$,

$$
i^{*} i_{*} \alpha=\alpha \cdot c_{1}\left(\mathcal{O}_{E}(E)\right)
$$

by the self-intersection formula for higher Chow groups, as recalled in Section 1. So $i^{*} i_{*}: \mathrm{CH}^{r}(E, n) \rightarrow \mathrm{CH}^{r+1}(E, n)$ is induced by $\delta_{*}\left(c_{1}\left(\mathcal{O}_{E}(E)\right)\right)$.

Consider the composition of maps ( $\pi_{F}$ and $\pi_{E}$ are the structure maps)

$$
\mathrm{CH}^{r}(p t, n) \stackrel{\pi_{F}^{*}}{\longrightarrow} \mathrm{CH}^{r}(F, n) \stackrel{i^{*} j_{*}}{\longrightarrow} \mathrm{CH}^{r+1}(E, n) \stackrel{\left(\pi_{E}\right)_{*}}{\longrightarrow} \mathrm{CH}^{r}(p t, n) .
$$

It is the multiplication by the integer $(E \cdot F)$. Indeed it is induced by the correspondence

$$
\left(\pi_{F} \times \pi_{E}\right)_{*} \delta_{*}(E \cap F)=(E \cdot F) \in \mathrm{CH}_{0}(p t)=\mathbb{Z}
$$

in case $E \neq F$ and by $\left(\pi_{F} \times \pi_{E}\right)_{*} \delta_{*}\left(c_{1}\left(\mathcal{O}_{E}(E)\right)\right)=(E \cdot E)$ in case $E=F$. (If $E \neq F$, the number $(E \cdot F)=1$ since $k$ is algebraically closed. But as we mentioned at the beginning of this section, one may assume $k$ arbitrary, in which case $(E \cdot F)$ is a positive integer.)

We go back to the assumption of Section 2. For the normal crossing divisor $E$, we studied its Chow cohomology and homology in Section 1. Let $a: E^{(0)} \rightarrow E$ be the canonical map, and $\tilde{\iota}=\iota \circ a: E^{(0)} \rightarrow X$. From the above argument we have: 
Lemma 3.1. The following square commutes:

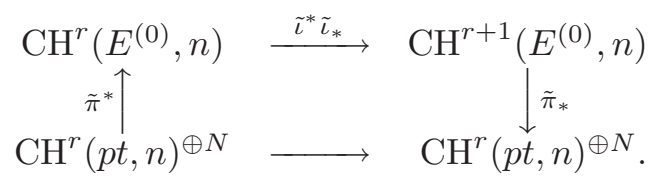

Here the lower horizontal map is given by the matrix $\left(E_{i} \cdot E_{j}\right)$, which is a $\mathbb{Q}$-isomorphism.

It follows the commutativity of the square

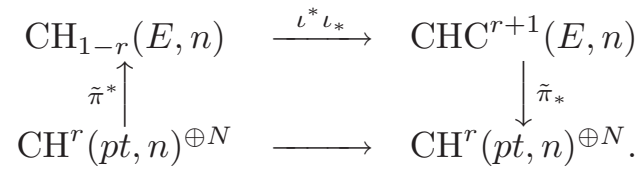

One still has a commutative square if one replaces $\mathrm{CH}_{1-r}(E, n)$ with $\widetilde{\mathrm{CH}}_{1-r}(E, n)$, and $\mathrm{CHC}^{r+1}(E, n)$ with $\widetilde{\mathrm{CHC}}^{r+1}(E, n)$.

Lemma 3.2. Assume that $E$ is a rational tree. (1) The map $\iota^{*}: \mathrm{CH}^{r}(X, n) \rightarrow$ $\widetilde{\mathrm{CHC}}^{r}(E, n)$ is surjective. So the long exact sequence of (2.1) splits into short exact sequences.

(2) The map $\iota_{*}: \widetilde{\mathrm{CH}}_{s}(E, n) \rightarrow \mathrm{CH}_{s}(X, n)$ is injective; the long exact sequence of (2.2) splits into short exact sequences.

Proof. (1) Since $\tilde{\pi}_{*}: \widetilde{\mathrm{CHC}}^{r}(E, n) \rightarrow \mathrm{CH}^{r-1}(p t, n)^{\oplus N}$ is an isomorphism by (1.4), it is enough to show that the composition $\tilde{\pi}_{*} \iota^{*}: \mathrm{CH}^{r}(X, n) \rightarrow \mathrm{CHC}^{r}(E, n) \rightarrow$ $\mathrm{CH}^{r-1}(p t, n)^{\oplus N}$ is surjective. But that is a consequence of Lemma 3.1. The proof of (2) is similar.

Theorem 3.3. Assume that $k$ is an algebraically closed field of characteristic zero, and let $S, X$, and $E$ be as in Section 2. Then the following conditions are equivalent:

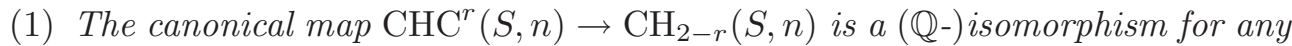
$r, n$.

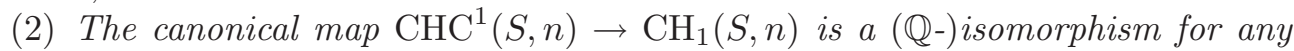
$n$.

(3) $E$ is a rational tree.

Proof. (1) clearly implies (2). We first show that (3) implies (1). If $E$ is a rational tree, by (3.2) there are exact sequences

$$
0 \rightarrow \mathrm{CHC}^{r}(S, n) \stackrel{p^{*}}{\longrightarrow} \mathrm{CH}^{r}(X, n) \stackrel{\iota^{*}}{\longrightarrow} \widetilde{\mathrm{CHC}^{r}}(E, n) \rightarrow 0
$$

and

$$
0 \rightarrow \widetilde{\mathrm{CH}}_{2-r}(E, n) \stackrel{\iota_{*}}{\longrightarrow} \mathrm{CH}_{2-r}(X, n) \stackrel{p_{*}}{\longrightarrow} \mathrm{CH}_{2-r}(S, n) \rightarrow 0
$$

where the middle terms are equal. 
In the commutative diagram of (3.1),

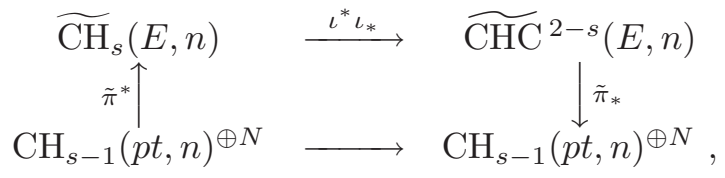

the lower horizontal map is a $(\mathbb{Q}$-)isomorphism, and the vertical maps are isomor-

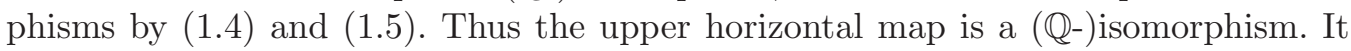
follows that the map $p_{*} p^{*}: \mathrm{CHC}^{r}(S, n) \rightarrow \mathrm{CH}_{2-r}(S, n)$ is an isomorphism.

We next show (2) implies (3). Since $\mathrm{CH}_{1}(S,-1)=0$, one has $\operatorname{CHC}^{1}(S,-1)=0$ and an exact sequence

$$
0 \rightarrow \mathrm{CHC}^{1}(S) \stackrel{p^{*}}{\longrightarrow} \mathrm{CH}^{1}(X) \stackrel{\iota^{*}}{\longrightarrow} \mathrm{CHC}^{1}(E) \rightarrow 0 .
$$

On the other hand by (2.2) there is an exact sequence

$$
0 \rightarrow \mathrm{CH}_{1}(E) \stackrel{\iota_{*}}{\longrightarrow} \mathrm{CH}_{1}(X) \stackrel{p_{*}}{\longrightarrow} \mathrm{CH}_{1}(S) \rightarrow 0 \text {. }
$$

Since $p_{*} p^{*}$ is an isomorphism, $\iota^{*} \iota_{*}: \mathrm{CH}_{1}(E) \rightarrow \mathrm{CHC}^{1}(E)$ is an isomorphism.

The group $\mathrm{CHC}^{1}(E)$ has a filtration as follows. By (1.3), there is a surjection $p^{*}: \mathrm{CHC}^{1}(E) \rightarrow \mathrm{CH}^{1}\left(E^{(0)}\right)$ with kernel $\left(H^{1}(\Gamma) \otimes k^{*}\right)_{\mathbb{Q}}$. Also the degree map deg : $\mathrm{CH}^{1}\left(E^{(0)}\right) \rightarrow \mathbb{Q}^{N}$ is a surjection with kernel $\bigoplus \operatorname{Pic}^{0}\left(E_{i}\right)_{\mathbb{Q}}$. But the composition

$$
\mathrm{CH}_{1}(E) \stackrel{\iota^{*} \iota_{*}}{\longrightarrow} \mathrm{CHC}^{1}(E) \stackrel{a^{*}}{\longrightarrow} \mathrm{CH}^{1}\left(E^{(0)}\right) \stackrel{\operatorname{deg}}{\longrightarrow} \mathbb{Q}^{N}
$$

is an isomorphism. Thus for $\iota^{*} \iota_{*}$ be an isomorphism, it is necessary that $\left(H^{1}(\Gamma) \otimes\right.$ $\left.k^{*}\right)_{\mathbb{Q}}=0$ and $\operatorname{Pic}^{0}\left(E_{i}\right)_{\mathbb{Q}}=0$ for each $i$. Since the characteristic of $k$ is zero, $k^{*}$ has a non-zero free subgroup, so the first condition implies $H^{1}(\Gamma)=0$. The condition $\operatorname{Pic}^{0}\left(E_{i}\right)_{\mathbb{Q}}=0$ implies $E_{i} \cong \mathbb{P}^{1}$. This concludes the proof.

Remark. (1) The assumption on the characteristic is necessary, since if $k$ is the algebraic closure of a finite field, then the group $k^{*} \otimes \mathbb{Q}$ is zero.

(2) If $k$ is the complex number field, the condition (3) is equivalent to the link of each singular point of $S$ being a rational homology sphere.

(3) There is a "motivic" version of the theorem where the Chow groups are replaced with mixed motives (see [Ham] for mixed motives of varieties). We will take this up elsewhere.

\section{Acknowledgments}

The author is very grateful to S.-I. Kimura, M. Tomari and K.-I. Watanabe for helpful discussions. He also thanks the referee who carefully read the manuscript and gave helpful comments.

\section{References}

[Bl 1] S. Bloch, Algebraic cycles and higher K-theory, Adv. Math. 61 (1986), 267-304.

[Bl 2] S. Bloch, The moving lemma for higher Chow groups, J. Algebraic Geom. 3 (1994), 537-568.

[Bl 3] S. Bloch, Some notes on elementary properties of higher chow groups, including functoriality properties and cubical chow groups, preprint on Bloch's home page, http://www.math.uchicago. edu/bloch.

[FV] E. Friedlander and V. Voevodsky, Bivariant cycle cohomology, Cycles, transfers, and motivic homology theories (by V. Voevodsky, A. Suslin, and E.M. Friedlander), Princeton University Press, Princeton, NJ, 2000. 
[Fu] W. Fulton, Intersection theory, Springer-Verlag, Berlin 1984.

[GNPP] F. Guillen, V. Navarro-Aznar, P. Pascual-Gainza and F. Puerta, Hyperresolutions cubiques et descente cohomologique, LNM 1335, Springer-Verlag, 1988.

[Ham] M. Hanamura, Mixed motives and algebraic cycles I, Math. Res. Lett. 2 (1995), 811-821. II, Invent. Math. 158 (2004), 105-179. III, Math. Res. Lett. 6 (1999), 61-82.

[Ha 2] M. Hanamura, Homological and cohomological motives of algebraic varieties, Invent. Math. 142 (2000), 319-349.

[Ha 3] M. Hanamura, Blow-ups and mixed motives, Tohoku Math. J., 63 (2011), 751-773.

[Ki] S.-I. Kimura, On the characterization of Alexander schemes, Compostio Math. 92 (1994), 273284.

[Ma] Y.I. Manin, Correspondences, motifs and monoidal transformations, Math. USSR Sbornik 6 (1968), 439-470.

$[\mathrm{Mu}] \mathrm{D}$. Mumford, The topology of normal singularities of an algebraic surface and a criterion for simplicity, Inst. Hautes Etudes Sci. Publ. Math. 9 (1961), 5-22.

[Vi] A. Vistoli, Alexander duality in intersection theory, Compos. Math. 70 (1989), 199-225.

Department of Mathematics, Tohoku University, Aramaki Aoba-ku, 980-8587, Sendai, JAPAN

E-mail address: hanamura@math.tohoku.ac.jp 
